\title{
Association of lifestyle factors like alcohol and tobacco consumption with semen abnormalities
}

Authors

\section{Shambhavi Tripathi ${ }^{1}$, Manish Kumar Verma ${ }^{2 *}$, Dushyant Singh Gaur ${ }^{3}$}

${ }^{1}$ Department of Pathology, Hind Institute of Medical Sciences, Mau, Ataria, Sitapur, Uttar Pradesh, India ${ }^{2}$ Department of Physiology, Dr Ram Manohar Lohia Institute of Medical Sciences, Lucknow, Uttar Pradesh, India

${ }^{3}$ Department of Pathology, Himalayan Institute of Medical Sciences, Dehradun, Uttarakhand, India

*Corresponding Author

Manish Kumar Verma

Department of Physiology, Dr Ram Manohar Lohia Institute of Medical Sciences, Lucknow, Uttar Pradesh, India

\begin{abstract}
Objective: To study the prevalence of semen abnormalities (Teratozoospermia, Asthenozoospermia and Oligozoospermia) amongst cases indulging in alcohol use and tobacco smoking and chewing.

Material \& Method: This study was carried out in Pathology Department, HIMS, Dehradun. Of the 220 cases reported over a period of 12 month, 30 cases each were selected for control group (A) and study groups- Alcohol users (B), Smokers (C), Tobacco chewers (D) and those with all three addiction $(E)$. Standard protocol as per WHO was used to study various semen parameters. Teratozoospermia was studied by two independent observers. Study groups were compared with controls and data was analysed.

Results: Teratozoospermia $(T)$ was the most frequently found variable $(n=127 / 174,72.8 \%)$. Asthenozoospermia (A) was the second most frequent semen variable $(n=113 / 174,65.8 \%)$, while oligozoospermia $(O)$ was present in $55.4 \%$ cases $(n=97 / 174)$. Asthenozoospermia was the most predominant finding in case with gutka addiction $(n=21 / 74)$, Teratozoospermia was the most predominant finding in case with all three addictions $(n=23 / 79)$.

Conclusion: Lifestyle factors like cigarette smoking and alcohol intake do produce significant negative impact on semen quality.
\end{abstract}

Keywords: Asthenozoospermia, Teratozoospermia, Oligozoospermia, Lifestyle factor.

\section{Introduction}

Semen quality in the adult males can be affected by number of environmental and lifestyle factors. An increasing trend in male fertility observed in recent years may be associated at least in part with these factors, which are compounded by a change in life style. ${ }^{(1)}$ It is undeniable that good quality semen is essential for reproductive success. A significant increase in the incidence of male infertility has been described in the international literature, raising questions about its causes. ${ }^{(2)}$ Factors associated with lifestyle choices appear to be linked to male subfertility. Several researchers have reported that alcohol and tobacco consumption, both by chewing and smoking, are by far the most commonly recognized factors that 
contribute to abnormalities in sperm morphology. (3)

Tobacco chewing and smoking habits in men has been linked to significant decrease in semen quality, particularly sperm morphology in moderate/heavy smokers. ${ }^{(4)}$ Alcohol consumption has been reported to produce progressive damage to sperm morphology and spermatogenesis. The situation is further aggravated when these habits of tobacco chewing / smoking and alcohol intake co-exist ${ }^{(5)}$. The detection of nicotine, and its major metabolite cotinine, in the seminal plasma of smokers showed that the tobacco compounds cross the blood-testis barrier and create a toxic environment for the spermatozoa. Toxic components in the cigarette smoke can disrupt the testicular microcirculation and cause DNA or chromosomal damage in germinal cells. ${ }^{(6)}$ Alcohol influences the Sertoli cell functions, probably by producing damage to some of the proteins required for sperm cell production that the Sertoli cells provide. Thus, alcohol induced reduction in levels of testosterone, LH and FSH not only hampers their normal morphological development and maturation of spermatozoa (producing significant teratozoospermia), it also slows down the sperm production by testicular germ cells (oligozoospermia), especially in heavy alcoholics. $^{(7)}$

\section{Aims and Objectives}

The aim of our study to examine semen parameters in relation to different life styles of adult men like tobacco chewing, smoking and drinking alcohol and to study the prevalence of different semen abnormalities such as teratozoospermia, azoospermia and asthenozoospermia.

\section{Materials and Methods}

This study was carried out in Pathology Department, HIMS, Dehradun. Of the 220 cases reported over a period of 12 month, 30 cases each were selected for control group (A) and study groups- Alcohol users (B), Smokers (C), Tobacco chewers (D) and those with all three addiction (E).
Inclusion Criteria: The control and the case will be selected from patients coming for semen analysis in department of Pathology.

\section{Control Group:}

Group A ( $n=30)$ : Strict non-alcohol consumer and non-tobacco chewer or smoker. Age less than 45 years.

\section{Study Groups}

1) Group B $(n=30)$ : Alcohol user but strict nonsmoker/non tobacco chewer

a) Mild alcohol consumer - those consuming $40 \mathrm{gm}$ or less alcohol per day

b) Moderate alcohol consumer - consuming 40-80gm alcohol per day

c) Heavy alcohol consumer - consuming more than 80 gm alcohol per day

$(40 \mathrm{gm}=60 \mathrm{ml}$ alcohol $-70 \%)$

2) Group $C(n=30)$ : Smoker who are strict non-alcohol consumer and non-tobacco chewer

a. Light smoker : $1-20$ cigarette per day

b. Moderate smoker : 21 - 40 cigarette per day

c. Heavy smoker: more than 40 cigarette per day

3) Group $D(n=30)$ : Tobacco chewer who are nonsmoker and non-alcohol consumer

4) Group $E(n=30)$ : Those consuming alcohol as well as tobacco (chewing/smoking)

5) Group $F(n=56)$ : Those consuming either alcohol and tobacco (smoker) or alcohol and tobacco(chewing) or tobacco(smoker) and tobacco(chewing).

Exclusion Criteria: Following males are excluded from study group those suffering from Azoospermia, ex-smoker, ex-alcoholic, extobacco chewer, history of prolonged medication, history of intake of herbal medicine / herbal tonic, history of occupational exposure to various chemicals or extremes of temperature, history of injury to testes, varicocele, hydrocele, undescended testes, vasectomy surgeries, history of pyospermia, hemospermia, chronic urinary tract infection, history of negative fluctose test. 


\section{Collection of Sample}

Sample was collected in the hospital by masturbation in a clean, wide mouthed plastic container non-toxic to the spermatozoa after minimum of 2 days of abstinence but not more than 7 days. Universal precautions were taken in handling the specimen.

All semen samples were analyzed for following semen parameters: liquefaction time, volume, viscosity, amorphous particulate matter, agglutination, motility, viability, sperm density and morphology as per recommended guidelines according to WHO manual. ${ }^{(8)}$ Theses parameters, when taken together, indicated the presence and absence of the three main semen abnormalities; asthenozoospermia, teratozoospermia and oligozoospermia.

\section{Microscopy}

Semen samples were examined for sperm density, sperm motility, sperm vitality, sperm morphology. Sperm morphology was studied on Papanicolaou stained smear, counting a minimum of 200 spermatozoa using oil-immersion lens. Sperm vitality was assessed in wet mount smears after supravital staining in aqueous eosin. ${ }^{(9)}$

\section{Statistical Analysis}

Interpretation and analysis of observation using SPSS software (Statistical Package for Social Services) version 22. Qualitative Data was expressed in term of frequency and percentage. Quantitative data expressed in term of mean \pm SD and mean percent. ANOVA was used to compare the mean of variables in different groups.

\section{Results}

In this study we include 220 males whose semen analysis was reported at Department of Pathology, HIMS, over a period of 12 months. Of these 220 males, the largest group of individuals fell in the age group of 26-35 years $(n=142 / 220,64.5 \%)$ followed by those in 16 to 25 years age group (46/220, 21\%) (Table1).
Table 1: Distribution of Cases according to Age $(\mathrm{n}=220$

\begin{tabular}{|l|c|c|}
\hline Age group (Yrs) & No. of Cases & Percentage \\
\hline $16-25$ & 46 & 21 \\
\hline $26-35$ & 142 & 64.5 \\
\hline $36-45$ & 32 & 14.5 \\
\hline
\end{tabular}

Majority of cases $(n=37,16.3 \%)$ are addicted to alcohol, smoking and gutka (SAG) combined and followed by an addiction to alcohol and smoking (SA) combined ( $\mathrm{n}=35,15.9 \%)$ (Figure 1)

Figure 1: Distribution of cases according to addiction



The overall frequency of these three common addictions, individual or in conjunction with other addictions is given in Table 2 . We found that alcohol consumption was the most common single addiction amongst cases studied $(n=116 / 190$, $61 \%$ ). Smoking alone, was observed among $60 \%$ $(n=114 / 190)$ individuals. Tobacco chewing habit was present in $46.2 \%(n=87 / 190)$ individuals. The non addicts (controls were not shown in this table).

Table 2: Total frequency of Addiction $(n=190 / 220)$

\begin{tabular}{|l|c|c|c|c|}
\hline $\begin{array}{l}\text { Type of } \\
\text { Addiction }\end{array}$ & Categories & $\begin{array}{c}\text { No. of } \\
\text { cases }\end{array}$ & Total & $\begin{array}{c}\text { Percenta } \\
\text { ge }\end{array}$ \\
\hline $\begin{array}{l}\text { Smoking } \\
\text { (S) }\end{array}$ & $\begin{array}{c}\text { S+SA+SG+S } \\
\text { AG }\end{array}$ & $\begin{array}{c}34+35+9 \\
+36\end{array}$ & $\begin{array}{c}114 / 1 \\
90\end{array}$ & 60 \\
\hline $\begin{array}{l}\text { Alcohol } \\
\text { (A) }\end{array}$ & $\begin{array}{c}\mathrm{A}+\mathrm{SA}+\mathrm{AG}+\mathrm{S} \\
\mathrm{AG}\end{array}$ & $\begin{array}{c}33+35+1 \\
2+36\end{array}$ & $\begin{array}{c}116 / 1 \\
90\end{array}$ & 61 \\
\hline Gutka(G) & $\begin{array}{c}\mathrm{G}+\mathrm{SG}+\mathrm{AG}+\mathrm{S} \\
\mathrm{AG}\end{array}$ & $\begin{array}{c}30+9+12 \\
+36\end{array}$ & $\begin{array}{c}87 / 19 \\
0\end{array}$ & 46.2 \\
\hline
\end{tabular}

Accordingly, teratozoospermia (T) was the most frequently found variable $(n=127 / 174,72.8 \%)$. 
Asthenozoospermia (A) was the second most frequent semen variable $(\mathrm{n}=113 / 174,65.8 \%)$, while oligozoospermia $(\mathrm{O})$ was present in $55.4 \%$ cases $(n=97 / 174)$. (Figure 2)

Figure 2: Total frequency of major semen variables

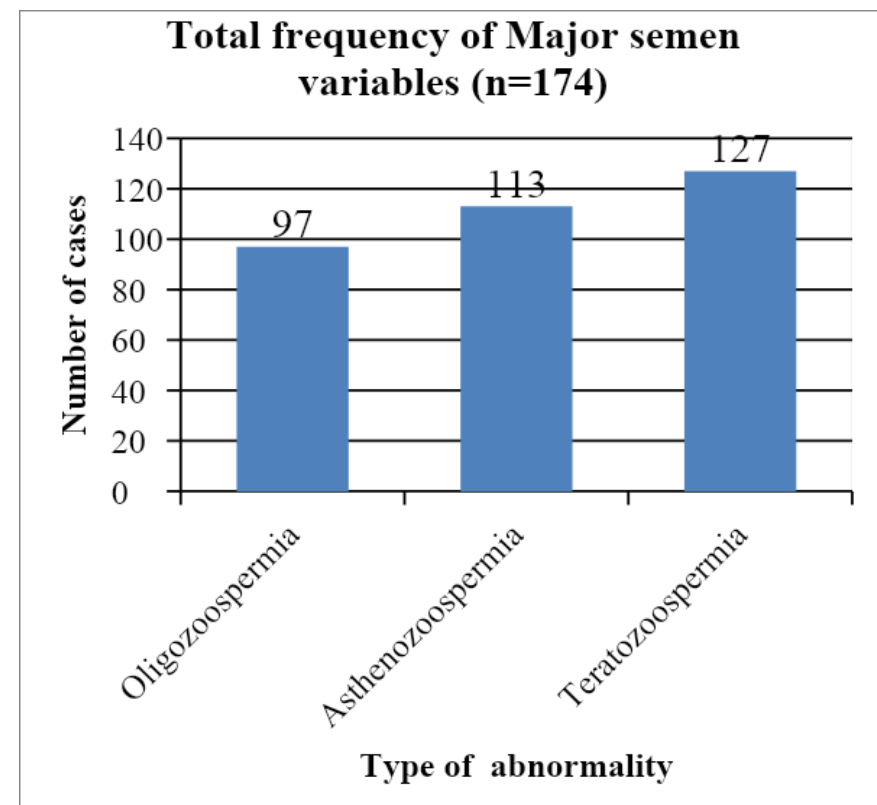

Asthenozoospermia was the most predominant finding in case with gutka addiction $(n=21 / 74)$, Teratozoospermia was the most predominant finding in case with all three addictions $(n=23 / 79)$. Controls $(n=30)$ and Azoospermics $(n=14)$ were not included in the analysis. (Figure 3 )

Figure 3: Frequency of abnormality in relation to type of addiction



\section{Discussion}

On correlating the three major semen variables $(\mathrm{O}, \mathrm{A}$ and $\mathrm{T})$ with the type of addiction, we found that asthenozoospermia was most common finding in subject addicted to gutka ( $n=21 / 57)$, Teratozoospermia was the most predominant finding in case addicted to alcohol $(n=20 / 50)$ and in cases with all three addictions $(n=23 / 64)($ fig 3$)$. This observation was in accordance with Gaur DS et al, where in tobacco smokers, the most dominant semen variable was asthenozoospermia (A), whereas in alcoholics Teratozoospermia (T) was the most dominant. ${ }^{(9)}$ Alcohol has been shown to have a detrimental effect on male reproductive hormones and semen quality. Alcohol consumption causes increase in $\beta$ endorphin levels that could be involved in testicular damage, inducing sperm apoptosis. Alcohol consumption disrupt nuclear maturity and DNA integrity. ${ }^{(10)}$ Alcohol intervene in the feedback mechanisms of hypothalamus-pituitarygonadal (HPG) axis resulting in impairment of production and secretion of leutinizing hormone (LH) and follicle stimulating hormone (FSH) leading to degeneration of sertoli cells. ${ }^{(9)}$ Chronic alcohol consumption resulted in high oxidative stress due to increased lipid peroxidation or due to decreased antioxidant mechanisms in testis. ${ }^{(11)}$ Thus, alcohol induced reduction in levels of testosterone, LH, FSH and increased level of oxidative stress not only hampers normal morphological development and maturation of spermatozoa, it also slows down the sperm production by testicular germ cells. A study by Goverde HJ et al on semen quality and frequency of smoking and alcohol consumption concluded that excessive alcohol consumption decreases the number of sperm with normal morphology. ${ }^{(12)}$ Similarly, Asare-Anane $\mathrm{H}$ et al studied effect of tobacco smoking on semen quality in 140 subjects and found that they were at higher risk of developing oligospermia, asthenozoospermia and teratozoospermia. ${ }^{(13)}$ A study by Kumar R et al concluded that tobacco chewing is strongly associated with a decrease in sperm quality and to 
a lesser extent with oligoasthenozoospermia or azoospermia. $^{(14)}$

Cigarette toxins increases the viscosity of seminal and other accessary glands secretions, causing the delayed liquefaction time and hence reducing forward linear progression of spermatozoa, manifesting as asthenozoospermia. Cigarette smoking causes oxidative stress due to excessive production of reactive oxygen species (ROS). ${ }^{(9)}$ ROS that are produced by spermatozoa for their special functions like capitation, the acrosome reaction and finally fertilization. Increased quantities of ROS have been responsible for DNA damage and defective membrane integrity of spermatozoa, thus producing a negative effect on the capability and morphology of spermatozoa ${ }^{(10)}$. In tobacco chewers, nicotine is absorbed through oral mucosa faster than smoking and residual toxins remain in circulation for a prolong period than in smokers. Concentration of nicotine and other substance in tobacco in epididymis disturbs its normal functioning particularly the activity of alpha-1,4 glycosidase enzyme. This inhibits secondary maturation of spermatozoa contributing to teratozoospermia. More over nicotine initiates huge ROS production leading to oxidative stress which causes DNA damage and contributes to teratozoospermia and oligozoospermia ${ }^{(15)}$.

\section{Conclusion}

Lifestyle factors like cigarette smoking and alcohol intake do produce significant negative impact on semen quality. Individuals being treated for infertility should abstain from these largely avoidable addictive lifestyle factors, alcohol and tobacco consumption.

\section{References}

1. Jurewicz J, Radwan M, Sobala W, Ligocka D, Radwan P, Bochenek M \& henke W. Lifestyle and semen quality: role of modifiable risk factors. Systems Biology in Reproductive Medicine, 2014; 60:1, 4351.
2. Gaur DS, Talekar MS, Pathak V. Effect of cigarette smoking on semen quality of infertile men. Singapore Med J. 2007; 48: 119-23

3. Bigazzi PE. Immunology of the male reproductive system. New York: Marcel Dekker; 1987.

4. Saaranen M, Suonio S, Kauhanen O, Saarikoski S. Cigarette smoking and semen quality in men of reproductive age. Andrologia. 1987; 19:670-6

5. Tamer M. Said, Ranga G, Agarwal A. Relationship between semen quality and tobacco chewing in men undergoing infertility evaluation. Fertility and Sterility. 2005;84(3): 649-53.

6. Chohan KR, Badawy SZA. Cigarette smoking impairs Sperm bioenergetics. International Braz J Urol. 2010; 36(1): 605.

7. Gordon GG, Altman K, Southern AL, Rubin E, Leiber CS. The effect of alcohol administration on sex hormone metabolism on normal men. N Engl J Med. 1976;295(15):793-7.

8. World health organization. Laboratory manual for examination of human semen and sperm-cervical mucus interaction. $3^{\text {rd }}$ ed. Newyork: Cambridge University Press, 1992.

9. Gaur DS, Talekar MS, Pathak V. Alcohol intake and cigarette smoking: Impact of two major lifestyle factors on male fertility. Indian J Pathol Microbiol. 2010; 53:35-40.

10. Sansone A, Dato CD, Angelis C, Menafra D, Pozza C, Pivonello R. Smoke, alcohol and drug addiction and male fertility. Reproductive Biology and Endocrinology. 2018; 16:3.

11. Albadri CT, Al Ani IM, Hiba HMA. Alcohol consumption and its effect on testicular structure and on sperm count and motility in parent mice and their offspring. 
The International Journal Malaysia. June 2013, vol 12:1.

12. Goverde HJ, Dekker HS, Janessen HJ, Bastiaans BA, Rolland R, Zielhuis GA. Semen quality and frequency of smoking and alcohol consumption- an explorative study. Int $\mathbf{J}$ Fertil Menopausal Stud 1995;40:135-8.

13. Asare-Anane H, Baninson SB, Ofori EK, Ateko RO, Bawah AT, Amanquah SD, Oppong SY, Gandau BBN, and Ziem JB. Tobacco smoking is associated with decreased semen quality. Reprod Health. 2016; 13: 90

14. Kumar R, Gautam G. Tobacco chewing and male infertility. Indian $\mathbf{J}$ Urol. 2006;22:161-2.

15. Sunanda P, Panda B, Dash C, Ray PK, Padhy RN, Routray P. Prevalence of abnormal spermatozoa in tobacco chewing sub-fertile males. J Hum Reprod Sci. 2014;7:136-42. 